\title{
SOME RESULTS ON EULER SUMS
}

\author{
Ce Xu, Jinfa Cheng
}

\begin{abstract}
In the paper, we develop an approach to evaluation of Euler sums that involve harmonic numbers and alternating harmonic numbers. We give explicit formulae for several classes of Euler sums in terms of Riemann zeta values and prove that the quadratic sums $S_{l^{2}, l}$ and cubic sums $S_{l^{3}, l}$ reduce to linear sums and polynomials in zeta values. The approach is based on constructive Power series and Cauchy product computations.
\end{abstract}

Keywords: Euler sums, Riemann zeta function, Cauchy product, power series.

\section{Introduction}

Harmonic numbers, alternating harmonic numbers and their generalizations are classically defined by

$$
H_{n}=\sum_{j=1}^{n} \frac{1}{j}, \quad \zeta_{n}(k)=\sum_{j=1}^{n} \frac{1}{j^{k}}, \quad L_{n}(k)=\sum_{j=1}^{n} \frac{(-1)^{j-1}}{j^{k}} .
$$

The subject of this paper is Euler sums, which are the infinite sums whose general term is a product of harmonic numbers and alternating harmonic numbers of index $n$ and a power of $n^{-1}$. So, we consider the Euler sums of the form

$$
\sum_{n=1}^{\infty} \frac{\prod_{i=1}^{m_{1}} \zeta_{n}^{q_{i}}\left(k_{i}\right) \prod_{j=1}^{m_{2}} L_{n}^{l_{j}}\left(h_{j}\right)}{n^{p}}, \quad \sum_{n=1}^{\infty} \frac{\prod_{i=1}^{m_{1}} \zeta_{n}^{q_{i}}\left(k_{i}\right) \prod_{j=1}^{m_{2}} L_{n}^{l_{j}}\left(h_{j}\right)(-1)^{n-1}}{n^{p}}
$$

where $m_{1}, m_{2}, q_{i}, k_{i}, h_{j}, l_{j}, p(p \geqslant 2)$ are positive integer. If $\sum_{i=1}^{m_{1}}\left(k_{i} q_{i}\right)+\sum_{j=1}^{m_{2}}\left(h_{j} l_{j}\right)+$ $p=C$ ( $C$ is a positive integer $)$, then we call it $C$-order Euler sums. Apart from the actual evaluation of the series, one of the main questions that one sets out to

Supported by Nature Science Fund of Fujian Province (grant no. 2011J01021) and Fundamental Research Funds for the Central Universities (grant no. 20720150006)

2010 Mathematics Subject Classification: primary: 11L99; secondary: 11M06 
solve is whether or not a given series can be expressed in terms of a linear rational combination of known constants. When this is the case, we say that the series is reducible to these values. It has been discovered in the course of the years that many Euler sums admit expressions involving finitely the zeta values, that is to say value of the Riemann zeta function,

$$
\zeta(s)=\sum_{n=1}^{\infty} \frac{1}{n^{s}}, \quad \operatorname{Re}(s)>1
$$

with the positive integers.

For a pair $(p, q)$ of positive integers with $p \geqslant 2$, the classical linear Euler sum is defined by

$$
S_{p, q}=\sum_{n=1}^{\infty} \frac{1}{n^{p}} \sum_{k=1}^{n} \frac{1}{k^{q}} .
$$

In 1742, Goldbach proposed to Euler the problem of expressing the $S_{p, q}$ in terms of values at positive integers of the Riemann zeta function $\zeta(s)$. Euler showed this problem in the case $p=1$ and gave a general formula for odd weight $p+q$ without any proof in 1775 .

Let $\pi=\left(\pi_{1}, \ldots, \pi_{k}\right)$ be a partition of integer $p$ into $k$ summands, so that $p=\pi_{1}+\cdots+\pi_{k}$ and $\pi_{1} \leqslant \pi_{2} \leqslant \cdots \leqslant \pi_{k}$. The classical nonlinear Euler sum of index $\pi, q$ is defined by (see [5])

$$
S_{\pi, q}=\sum_{n=1}^{\infty} \frac{\zeta_{n}\left(\pi_{1}\right) \zeta_{n}\left(\pi_{2}\right) \cdots \zeta_{n}\left(\pi_{k}\right)}{n^{q}}
$$

the quantity $\pi_{1}+\cdots+\pi_{k}+q$ being called the weight and the quantity $k$ being the degree. As usual, repeated summands in partitions are indicated by powers, so that for instance

$$
S_{1^{2} 2^{3} 4, q}=S_{112224, q}=\sum_{n=1}^{\infty} \frac{H_{n}^{2} \zeta_{n}^{3}(2) \zeta_{n}(4)}{n^{q}} .
$$

The relationship between the values of the Riemann zeta function and Euler sums has been studied by many authors, for example see [1-5,7]. Philippe Flajolet and Bruno Salvy (see[5]) made use of contour integral to obtain some representation of $\sum_{n=1}^{\infty} \frac{\zeta_{n}(q)}{n^{p}}, \sum_{n=1}^{\infty} \frac{H_{n}^{q}}{n^{p}}$ by Riemann zeta function. In [1], David H. Bailey, Jonathan M. Borwein and Roland Girgensohn considered sums of the form $\sum_{n=1}^{\infty} \frac{L_{n}^{q}(1)}{n^{p}}, \sum_{n=1}^{\infty} \frac{L_{n}^{q}(1)}{n^{p}}(-1)^{n-1}$, where $p$ and $q$ are positive integers. In [7], Ping SUN made use of probabilistic and combinatiorial methods to obtain some representation of sums of the form $\sum_{n=1}^{\infty} \frac{\prod_{i=1}^{m_{1}} \zeta_{n}^{q_{i}}\left(k_{i}\right)}{n^{p}}$ by Riemann zeta function. But so far, no one has solved the following Euler sums,

$$
\sum_{n=1}^{\infty} \frac{\zeta_{n}^{2}(3)}{n^{3}}, \sum_{n=1}^{\infty} \frac{L_{n}(1) L_{n}(3)}{n+1}(-1)^{n-1}, \sum_{n=1}^{\infty} \frac{L_{n}(1) \zeta_{n}(3)}{n+1}(-1)^{n-1} .
$$


This paper develops an approach to the evaluation of Euler sums. The main purpose of this paper is to evaluation of some quadratic Euler sums that involve harmonic numbers and alternating harmonic numbers. We give explicit formulae for several classes of Euler sums in terms of Riemann zeta values and analytic value of (1.4) and establish the following important equations. For an integer $l \geqslant 2$, we have

$$
\begin{gathered}
S_{l^{2}, l}=\sum_{n=1}^{\infty} \frac{\zeta_{n}^{2}(l)}{n^{l}}=\sum_{n=1}^{\infty} \frac{\zeta_{n}(l)}{n^{2 l}}+\frac{1}{3}\left(\zeta^{3}(l)-\zeta(3 l)\right) \\
\sum_{n=1}^{\infty} \frac{L_{n}^{2}(l)(-1)^{n-1}}{n^{l}}=\sum_{n=1}^{\infty} \frac{L_{n}(l)}{n^{2 l}}+\frac{1}{3}\left(L^{3}(l)-L(3 l)\right)
\end{gathered}
$$

where $\zeta(s)=\sum_{n=1}^{\infty} \frac{1}{n^{s}}, L(s)=\sum_{n=1}^{\infty} \frac{(-1)^{n-1}}{n^{s}}$. and we prove that the cubic sums $S_{l^{3}, l}$ reduce to linear sums and polynomials in zeta values.

From (1.5) we know that the quadratic sums $S_{l^{2}, l}$ reduce to linear sums and polynomials in zeta values. A general class of quadratic sums $S_{p_{1} p_{2}, q}\left(1<p_{1}\right.$, $\left.p_{2}, q \in Z\right)$ was studied by Flajolet and Salvy [5]. $S_{p_{1} p_{2}, q}\left(1<p_{1}, p_{2}, q \in Z\right)$ are reducible to linear sums, but $p_{1}, p_{2}, q$ should satisfy the condition $p_{1}+p_{2}+q$ is even.

\section{Main theorems and the proof}

Theorem 2.1. For $l_{1}, l_{2}, m_{1}, m_{2} \geqslant 2$ and $l_{1}, l_{2}, m_{1}, m_{2} \in Z^{+}$, we have

$$
\begin{aligned}
\zeta^{m_{1}}\left(l_{1}\right) \zeta^{m_{2}}\left(l_{2}\right)= & \sum_{k=1}^{\infty}\left\{\sum_{j_{1}=1}^{m_{1}-1}\left(\begin{array}{c}
m_{1} \\
j_{1}
\end{array}\right) \frac{\zeta_{k}^{j_{1}}\left(l_{1}\right)}{(k+1)^{l_{2} m_{2}+\left(m_{1}-j_{1}\right) l_{1}}}\right. \\
& \left.+\sum_{j_{2}=1}^{m_{2}-1}\left(\begin{array}{c}
m_{2} \\
j_{2}
\end{array}\right) \frac{\zeta_{k}^{j_{2}}\left(l_{2}\right)}{(k+1)^{l_{1} m_{1}+\left(m_{2}-j_{2}\right) l_{2}}}\right\} \\
& +\sum_{k=1}^{\infty}\left\{\frac{\zeta_{k}^{m_{2}}\left(l_{2}\right)}{\left.(k+1)^{m_{1} l_{1}}+\frac{\zeta_{k}^{m_{1}}\left(l_{1}\right)}{(k+1)^{m_{2} l_{2}}}\right\}+\zeta\left(m_{1} l_{1}+m_{2} l_{2}\right)}\right. \\
& +\sum_{k=1}^{\infty} \sum_{j_{1}=1}^{m_{1}-1} \sum_{j_{2}=1}^{m_{2}-1}\left(\begin{array}{c}
m_{1} \\
j_{1}
\end{array}\right)\left(\begin{array}{c}
m_{2} \\
j_{2}
\end{array}\right) \frac{\zeta_{k}^{j_{1}}\left(l_{1}\right) \zeta_{k}^{j_{2}}\left(l_{2}\right)}{(k+1)^{\left(m_{1}-j_{1}\right) l_{1}+\left(m_{2}-j_{2}\right) l_{2}}} \\
& +\sum_{k=1}^{\infty}\left\{\sum_{j_{1}=1}^{m_{1}-1}\left(\begin{array}{c}
m_{1} \\
j_{1}
\end{array}\right) \frac{\zeta_{k}^{j_{1}}\left(l_{1}\right) \zeta_{k}^{m_{2}}\left(l_{2}\right)}{(k+1)^{\left(m_{1}-j_{1}\right) l_{1}}}\right. \\
& \left.+\sum_{j_{2}=1}^{m_{2}-1}\left(\begin{array}{c}
m_{2} \\
j_{2}
\end{array}\right) \frac{\zeta_{k}^{j_{2}}\left(l_{2}\right) \zeta_{k}^{m_{1}}\left(l_{1}\right)}{(k+1)^{\left(m_{2}-j_{2}\right) l_{2}}}\right\}
\end{aligned}
$$


Proof. First, constructing function $y=\sum_{n=1}^{\infty} \zeta_{n}^{m_{1}}\left(l_{1}\right) \zeta_{n}^{m_{2}}\left(l_{2}\right) x^{n}(|x|<1)$. Using the definition of the harmonic numbers $\zeta_{n}(k)(1.1)$ we have

$$
y=x+\sum_{n=1}^{\infty}\left(\zeta_{n}\left(l_{1}\right)+\frac{1}{(n+1)^{l_{1}}}\right)^{m_{1}}\left(\zeta_{n}\left(l_{2}\right)+\frac{1}{(n+1)^{l_{2}}}\right)^{m_{2}} x^{n+1} .
$$

Using the Newton binomial expansion, we get

$$
\begin{aligned}
y= & x y+\sum_{n=1}^{\infty} \frac{x^{n}}{n^{l_{1} m_{1}+l_{2} m_{2}}}+\sum_{n=1}^{\infty}\left\{\frac{\zeta_{n}^{m_{2}}\left(l_{2}\right)}{(n+1)^{m_{1} l_{1}}}+\frac{\zeta_{n}^{m_{1}}\left(l_{1}\right)}{(n+1)^{m_{2} l_{2}}}\right\} x^{n+1} \\
& +\sum_{n=1}^{\infty}\left\{\sum_{j_{1}=1}^{m_{1}-1}\left(\begin{array}{c}
m_{1} \\
j_{1}
\end{array}\right) \frac{\zeta_{n}^{j_{1}}\left(l_{1}\right)}{(n+1)^{l_{2} m_{2}+\left(m_{1}-j_{1}\right) l_{1}}}\right. \\
& \left.+\sum_{j_{2}=1}^{m_{2}-1}\left(\begin{array}{c}
m_{2} \\
j_{2}
\end{array}\right) \frac{\zeta_{n}^{j_{2}}\left(l_{2}\right)}{(n+1)^{l_{1} m_{1}+\left(m_{2}-j_{2}\right) l_{2}}}\right\} x^{n+1} \\
& +\sum_{n=1}^{\infty} \sum_{j_{1}=1}^{m_{1}-1} \sum_{j_{2}=1}^{m_{2}-1}\left(\begin{array}{c}
m_{1} \\
j_{1}
\end{array}\right)\left(\begin{array}{c}
m_{2} \\
j_{2}
\end{array}\right) \frac{\zeta_{n}^{j_{1}}\left(l_{1}\right) \zeta_{n}^{j_{2}}\left(l_{2}\right)}{(n+1)^{\left(m_{1}-j_{1}\right) l_{1}+\left(m_{2}-j_{2}\right) l_{2}}} x^{n+1} \\
& +\sum_{n=1}^{\infty}\left\{\sum_{j_{1}=1}^{m_{1}-1}\left(\begin{array}{c}
m_{1} \\
j_{1}
\end{array}\right) \frac{\zeta_{n}^{j_{1}}\left(l_{1}\right) \zeta_{n}^{m_{2}}\left(l_{2}\right)}{(n+1)^{\left(m_{1}-j_{1}\right) l_{1}}}+\sum_{j_{2}=1}^{m_{2}-1}\left(\begin{array}{c}
m_{2} \\
j_{2}
\end{array}\right) \frac{\zeta_{n}^{j_{2}}\left(l_{2}\right) \zeta_{n}^{m_{1}}\left(l_{1}\right)}{(n+1)^{\left(m_{2}-j_{2}\right) l_{2}}}\right\} x^{n+1} .
\end{aligned}
$$

First, Move $x y$ from the right to the left, then multiply both sides by $(1-x)^{-1}$. Using formula $(1-x)^{-1}=\sum_{n=1}^{\infty} x^{n-1}, x \in(-1,1)$ and Cauchy product formula, then equate the coefficient of $x^{n+1}$ on both sides, we get

$$
\begin{aligned}
\zeta_{n+1}^{m_{1}}\left(l_{1}\right) \zeta_{n+1}^{m_{2}} & \left(l_{2}\right)=\sum_{k=1}^{n}\left\{\sum_{j_{1}=1}^{m_{1}-1}\left(\begin{array}{c}
m_{1} \\
j_{1}
\end{array}\right) \frac{\zeta_{k}^{j_{1}}\left(l_{1}\right)}{(k+1)^{l_{2} m_{2}+\left(m_{1}-j_{1}\right) l_{1}}}\right. \\
& \left.+\sum_{j_{2}=1}^{m_{2}-1}\left(\begin{array}{c}
m_{2} \\
j_{2}
\end{array}\right) \frac{\zeta_{k}^{j_{2}}\left(l_{2}\right)}{(k+1)^{l_{1} m_{1}+\left(m_{2}-j_{2}\right) l_{2}}}\right\} \\
& +\sum_{k=1}^{n}\left\{\frac{\zeta_{k}^{m_{2}}\left(l_{2}\right)}{\left.(k+1)^{m_{1} l_{1}}+\frac{\zeta_{k}^{m_{1}}\left(l_{1}\right)}{(k+1)^{m_{2} l_{2}}}\right\}+\zeta_{n+1}\left(m_{1} l_{1}+m_{2} l_{2}\right)}\right. \\
& +\sum_{k=1}^{n} \sum_{j_{1}=1}^{m_{1}-1} \sum_{j_{2}=1}^{m_{2}-1}\left(\begin{array}{c}
m_{1} \\
j_{1}
\end{array}\right)\left(\begin{array}{c}
m_{2} \\
j_{2}
\end{array}\right) \frac{\zeta_{k}^{j_{1}}\left(l_{1}\right) \zeta_{k}^{j_{2}}\left(l_{2}\right)}{(k+1)^{\left(m_{1}-j_{1}\right) l_{1}+\left(m_{2}-j_{2}\right) l_{2}}} \\
& +\sum_{k=1}^{n}\left\{\sum_{j_{1}=1}^{m_{1}-1}\left(\begin{array}{c}
m_{1} \\
j_{1}
\end{array}\right) \frac{\zeta_{k}^{j_{1}}\left(l_{1}\right) \zeta_{k}^{m_{2}}\left(l_{2}\right)}{(k+1)^{\left(m_{1}-j_{1}\right) l_{1}}}+\sum_{j_{2}=1}^{m_{2}-1}\left(\begin{array}{c}
m_{2} \\
j_{2}
\end{array}\right) \frac{\zeta_{k}^{j_{2}}\left(l_{2}\right) \zeta_{k}^{m_{1}}\left(l_{1}\right)}{(k+1)^{\left(m_{2}-j_{2}\right) l_{2}}}\right\} .
\end{aligned}
$$

Letting $n \rightarrow \infty$ gives (2.1). 
Theorem 2.2. For $l_{1}, l_{2}, m_{2} \geqslant 2$ and $l_{1}, l_{2}, m_{2} \in Z^{+}$, we have

$$
\begin{aligned}
\zeta\left(l_{1}\right) \zeta^{m_{2}}\left(l_{2}\right)= & \sum_{k=1}^{\infty} \sum_{j_{2}=1}^{m_{2}-1}\left(\begin{array}{c}
m_{2} \\
j_{2}
\end{array}\right) \frac{\zeta_{k}^{j_{2}}\left(l_{2}\right) \zeta_{k}\left(l_{1}\right)}{(k+1)^{\left(m_{2}-j_{2}\right) l_{2}}} \\
& +\sum_{k=1}^{\infty} \sum_{j_{2}=1}^{m_{2}-1}\left(\begin{array}{c}
m_{2} \\
j_{2}
\end{array}\right) \frac{\zeta_{k}^{j_{2}}\left(l_{2}\right)}{(k+1)^{\left(m_{2}-j_{2}\right) l_{2}+l_{1}}} \\
& +\sum_{k=1}^{\infty} \frac{\zeta_{k}^{m_{2}}\left(l_{2}\right)}{(k+1)^{l_{1}}}+\zeta\left(l_{1}+m_{2} l_{2}\right)+\sum_{k=1}^{\infty} \frac{\zeta_{k}\left(l_{1}\right)}{(k+1)^{m_{2} l_{2}}} .
\end{aligned}
$$

Proof. Similarly to Theorem 2.1, constructing function $y=\sum_{n=1}^{\infty} \zeta_{n}\left(l_{1}\right) \zeta_{n}^{m_{2}}\left(l_{2}\right) x^{n}$ $(|x|<1)$, we deduce that Theorem 2.2.

Theorem 2.3. For $l_{1}, l_{2}, m_{1}, m_{2} \geqslant 2$ with $l_{1}, l_{2}, m_{1}, m_{2} \in Z^{+}$and $|x| \leqslant 1$, we get

$$
\begin{aligned}
& \zeta_{n+1}^{m_{1}}\left(l_{1}, x\right) \zeta_{n+1}^{m_{2}}\left(l_{2}, x\right)=\sum_{k=1}^{n} \frac{\zeta_{k}^{m_{1}}\left(l_{1}, x\right) x^{k m_{2}}}{(k+1)^{m_{2} l_{2}}} \\
& +\sum_{k=1}^{n} \sum_{j_{2}=1}^{m_{2}-1}\left(\begin{array}{c}
m_{2} \\
j_{2}
\end{array}\right) \frac{\zeta_{k}^{m_{1}}\left(l_{1}, x\right) \zeta_{k}^{j_{2}}\left(l_{2}, x\right) x^{k\left(m_{2}-j_{2}\right)}}{(k+1)^{\left(m_{2}-j_{2}\right) l_{2}}} \\
& +\sum_{k=1}^{n} \frac{\zeta_{k}^{m_{2}}\left(l_{2}, x\right) x^{k m_{1}}}{(k+1)^{m_{1} l_{1}}}+\sum_{k=1}^{n} \sum_{j_{2}=1}^{m_{2}-1}\left(\begin{array}{c}
m_{2} \\
j_{2}
\end{array}\right) \frac{\zeta_{k}^{j_{2}}\left(l_{2}, x\right) x^{k\left(m_{1}+m_{2}-j_{2}\right)}}{(k+1)^{m_{1} l_{1}+\left(m_{2}-j_{2}\right) l_{2}}} \\
& +\sum_{k=1}^{n} \sum_{j_{1}=1}^{m_{1}-1}\left(\begin{array}{c}
m_{1} \\
j_{1}
\end{array}\right) \frac{\zeta_{k}^{m_{2}}\left(l_{2}, x\right) \zeta_{k}^{j_{1}}\left(l_{1}, x\right) x^{k\left(m_{1}-j_{1}\right)}}{(k+1)^{\left(m_{1}-j_{1}\right) l_{1}}}+\sum_{k=0}^{n} \frac{x^{k\left(m_{1}+m_{2}\right)}}{(k+1)^{m_{1} l_{1}+m_{2} l_{2}}} \\
& +\sum_{k=1}^{n} \sum_{j_{1}=1}^{m_{1}-1} \sum_{j_{2}=1}^{m_{2}-1}\left(\begin{array}{c}
m_{1} \\
j_{1}
\end{array}\right)\left(\begin{array}{c}
m_{2} \\
j_{2}
\end{array}\right) \frac{\zeta_{k}^{j_{1}}\left(l_{1}, x\right) \zeta_{k}^{j_{2}}\left(l_{2}, x\right) x^{k\left(m_{1}+m_{2}-j_{1}-j_{2}\right)}}{(k+1)^{\left(m_{1}-j_{1}\right) l_{1}+\left(m_{2}-j_{2}\right) l_{2}}} \\
& +\sum_{k=1}^{n} \sum_{j_{1}=1}^{m_{1}-1}\left(\begin{array}{c}
m_{1} \\
j_{1}
\end{array}\right) \frac{\zeta_{k}^{j_{1}}\left(l_{1}, x\right) x^{k\left(m_{1}+m_{2}-j_{1}\right)}}{(k+1)^{m_{2} l_{2}+\left(m_{1}-j_{1}\right) l_{1}}} .
\end{aligned}
$$

If $m_{1}=1, m_{2} \geqslant 2$, we obtain

$$
\begin{aligned}
\zeta_{n+1}\left(l_{1}, x\right) \zeta_{n+1}^{m_{2}}\left(l_{2}, x\right)=\sum_{k=1}^{n} \frac{\zeta_{k}\left(l_{1}, x\right) x^{k m_{2}}}{(k+1)^{m_{2} l_{2}}} \\
\quad+\sum_{k=1}^{n} \sum_{j_{2}=1}^{m_{2}-1}\left(\begin{array}{c}
m_{2} \\
j_{2}
\end{array}\right) \frac{\zeta_{k}\left(l_{1}, x\right) \zeta_{k}^{j_{2}}\left(l_{2}, x\right) x^{k\left(m_{2}-j_{2}\right)}}{(k+1)^{\left(m_{2}-j_{2}\right) l_{2}}}+\sum_{k=1}^{n} \frac{\zeta_{k}^{m_{2}}\left(l_{2}, x\right) x^{k}}{(k+1)^{l_{1}}} \\
\quad+\sum_{k=1}^{n} \sum_{j_{2}=1}^{m_{2}-1}\left(\begin{array}{c}
m_{2} \\
j_{2}
\end{array}\right) \frac{\zeta_{k}^{j_{2}}\left(l_{2}, x\right) x^{k\left(1+m_{2}-j_{2}\right)}}{(k+1)^{l_{1}+\left(m_{2}-j_{2}\right) l_{2}}}+\sum_{k=0}^{n} \frac{x^{k\left(1+m_{2}\right)}}{(k+1)^{l_{1}+m_{2} l_{2}}} .
\end{aligned}
$$

where $\zeta_{n}\left(l_{1}, x\right)=1+\frac{x}{2^{l_{1}}}+\cdots+\frac{x^{n-1}}{n^{l_{1}}}, \zeta_{n}\left(l_{2}, x\right)=1+\frac{x}{2^{l_{2}}}+\cdots+\frac{x^{n-1}}{n^{l_{2}}}$. 
Proof. Similarly to Theorem 2.1 and 2.2, constructing function

$$
z=\sum_{n=1}^{\infty} \zeta_{n}^{m_{1}}\left(l_{1}, x\right) \zeta_{n}^{m_{2}}\left(l_{2}, x\right) y^{n}
$$

we deduce that Theorem 2.3. Obviously, Theorems 2.1 and 2.2 are special cases of Theorem 2.3.

Taking $m_{1}=m_{2}=2$ in $(2.1)$, we obtain

Corollary 2.4. For $l_{1}, l_{2} \geqslant 2$ and $l_{1}, l_{2} \in Z^{+}$, we have the relation

$$
\begin{aligned}
\zeta^{2}\left(l_{1}\right) \zeta^{2}\left(l_{2}\right)= & \sum_{n=1}^{\infty}\left\{\frac{\zeta_{n}^{2}\left(l_{2}\right)}{(n+1)^{2 l_{1}}}+\frac{\zeta_{n}^{2}\left(l_{1}\right)}{(n+1)^{2 l_{2}}}\right\} \\
& +2 \sum_{n=1}^{\infty}\left\{\frac{\zeta_{n}\left(l_{1}\right) \zeta_{n}^{2}\left(l_{2}\right)}{(n+1)^{l_{1}}}+\frac{\zeta_{n}\left(l_{1}\right)}{(n+1)^{2 l_{2}+l_{1}}}\right\} \\
& +\zeta\left(2 l_{1}+2 l_{2}\right)+4 \sum_{n=1}^{\infty} \frac{\zeta_{n}\left(l_{1}\right) \zeta_{n}\left(l_{2}\right)}{(n+1)^{l_{2}+l_{1}}} \\
& +2 \sum_{n=1}^{\infty}\left\{\frac{\zeta_{n}^{2}\left(l_{1}\right) \zeta_{n}\left(l_{2}\right)}{(n+1)^{l_{2}}}+\frac{\zeta_{n}\left(l_{2}\right)}{(n+1)^{2 l_{1}+l_{2}}}\right\} .
\end{aligned}
$$

Let $m_{1}=m_{2}=2, l_{1}=l_{2}=l$ in $(2.5)$, then

$$
\zeta^{4}(l)=\zeta(4 l)+6 \sum_{n=1}^{\infty} \frac{\zeta_{n}^{2}(l)}{(n+1)^{2 l}}+4 \sum_{n=1}^{\infty} \frac{\zeta_{n}^{3}(l)}{(n+1)^{l}}+4 \sum_{n=1}^{\infty} \frac{\zeta_{n}(l)}{(n+1)^{3 l}} .
$$

Taking $m_{2}=2$ in (2.2), we obtain

Corollary 2.5. For $l_{1}, l_{2} \geqslant 2$ and $l_{1}, l_{2} \in Z^{+}$, we have

$$
\begin{aligned}
\zeta\left(l_{1}\right) \zeta^{2}\left(l_{2}\right)= & \zeta\left(l_{1}+2 l_{2}\right)+2 \sum_{n=1}^{\infty} \frac{\zeta_{n}\left(l_{1}\right) \zeta_{n}\left(l_{2}\right)}{(n+1)^{l_{2}}}+2 \sum_{n=1}^{\infty} \frac{\zeta_{n}\left(l_{2}\right)}{(n+1)^{l_{1}+l_{2}}} \\
& +\sum_{n=1}^{\infty} \frac{\zeta_{n}^{2}\left(l_{2}\right)}{(n+1)^{l_{1}}}+\sum_{n=1}^{\infty} \frac{\zeta_{n}\left(l_{1}\right)}{(n+1)^{2 l_{2}}} .
\end{aligned}
$$

Let $m_{2}=2, l_{1}=l_{2}=l$ in $(2.7)$, we get

$$
\zeta^{3}(l)=\zeta(3 l)+3 \sum_{n=1}^{\infty} \frac{\zeta_{n}^{2}(l)}{(n+1)^{l}}+3 \sum_{n=1}^{\infty} \frac{\zeta_{n}(l)}{(n+1)^{2 l}} .
$$

Remark. Noting that the Riemann zeta function $\zeta^{3}(l)$, which can be rewritten as

$$
\zeta^{3}(l)=1+\sum_{n=1}^{\infty}\left(\zeta_{n+1}^{3}(l)-\zeta_{n}^{3}(l)\right)
$$


Then using the binomial theorem, we have

$$
\zeta_{n+1}^{3}(l)=\zeta_{n}^{3}(l)+3 \frac{\zeta_{n}^{2}(l)}{(n+1)^{l}}+3 \frac{\zeta_{n}(l)}{(n+1)^{2 l}}+\frac{1}{(n+1)^{3 l}} .
$$

Loading (2.10) in (2.9), we obtain (2.8).

From Theorem 2.3, let $m_{2}=2, l_{1}=l_{2}=l, n \rightarrow \infty$, we obtain

Corollary 2.6. For $l \geqslant 2, l \in Z^{+}$and $x \in[-1,1)$, we have

$$
L i_{l}^{3}(x)=3 \sum_{n=1}^{\infty} \frac{\zeta_{n}^{2}(l, x)}{(n+1)^{l}} x^{n+3}+3 \sum_{n=1}^{\infty} \frac{\zeta_{n}(l, x)}{(n+1)^{2 l}} x^{2 n+3}+L i_{3 l}\left(x^{3}\right),
$$

where $\operatorname{Li}_{l}(x)=\sum_{n=1}^{\infty} \frac{x^{n}}{n^{l}}$.

Taking $x=1, x=-1$ in (2.11) we obtain (1.5)(1.6). Taking $m_{2}=m-1, l_{1}=$ $l_{2}=1, n \rightarrow \infty$ in (2.4) and combining $L i_{1}(x)=-\ln (1-x)$, we obtain

Corollary 2.7. For $m \geqslant 2, l \in Z^{+}$and $x \in[-1,1)$, we obtain

$$
\begin{aligned}
& \ln ^{m}\left(\frac{1}{1-x}\right) \\
= & \left\{\sum_{n=1}^{\infty} \frac{x^{n m}}{n^{m}}+\sum_{j=1}^{m-1}\left(\begin{array}{c}
m \\
j
\end{array}\right) \sum_{n=1}^{\infty}\left(1+\frac{x}{2}+\cdots+\frac{x^{n-1}}{n}\right)^{j}\left(\frac{x^{n}}{n+1}\right)^{m-j} x^{m}\right\},
\end{aligned}
$$

where $x \in[-1,1)$.

In the same manner we obtain the more general identity

$$
\begin{aligned}
& L i_{l}^{m}(x) \\
& =\left\{\sum_{n=1}^{\infty} \frac{x^{n m}}{n^{l m}}+\sum_{j=1}^{m-1}\left(\begin{array}{c}
m \\
j
\end{array}\right) \sum_{n=1}^{\infty}\left(1+\frac{x}{2^{l}}+\cdots+\frac{x^{n-1}}{n^{l}}\right)^{j}\left(\frac{x^{n}}{(n+1)^{l}}\right)^{m-j} x^{m}\right\} .
\end{aligned}
$$

Lemma 2.8 ([6]). For $l \geqslant 2, l \in Z^{+}$and $x \in[-1,1)$, we have for $k \geqslant 0$,

$$
\sum_{n=k}^{\infty} S(n, k) \frac{t^{n}}{n !}=\ln ^{k}\left(\frac{1}{1-t}\right) / k !
$$

where $S(n, k)$ is Stirling numbers of the first kind and

$$
\begin{gathered}
S(n, 1)=(n-1) !, S(n, 2)=(n-1) ! H_{n-1}, S(n, 3)=\frac{(n-1) !}{2 !}\left[H_{n-1}^{2}-\zeta_{n}(2)\right], \\
S(n, 4)=\frac{(n-1) !}{3 !}\left[H_{n-1}^{3}-3 H_{n-1} \zeta_{n}(2)+2 \zeta_{n-1}(3)\right] .
\end{gathered}
$$


Taking $k=2,3,4$ in $(2.13)$ yields

\section{Corollary 2.9.}

$$
\begin{aligned}
& \ln ^{2}(1-x)=2 \sum_{n=1}^{\infty} \frac{H_{n} x^{n+1}}{n+1}, \ln ^{3}(1-x)=3 \sum_{n=1}^{\infty} \frac{\left(\zeta_{n}(2)-H_{n}^{2}\right) x^{n+1}}{n+1}, \\
& \ln ^{4}(1-x)=4 \sum_{n=1}^{\infty} \frac{\left(H_{n}^{3}-3 H_{n} \zeta_{n}(2)+2 \zeta_{n}(3)\right) x^{n+1}}{n+1},
\end{aligned}
$$

where $x \in[-1,1)$.

\section{Representation of Euler sums by Riemann zeta function}

Lemma 3.1 ([5]). For an odd weight $m=p+q$, the Euler sums are reducible to zeta values,

$$
\begin{aligned}
\sum_{n=1}^{\infty} \frac{\zeta_{n}(p)}{n^{q}}= & \zeta(m)\left(\frac{1}{2}-\frac{(-1)^{p}}{2}\left(\begin{array}{c}
m-1 \\
p
\end{array}\right)-\frac{(-1)^{p}}{2}\left(\begin{array}{c}
m-1 \\
q
\end{array}\right)\right) \\
& +(-1)^{p} \sum_{k=1}^{[p / 2]}\left(\begin{array}{c}
m-2 k-1 \\
q-1
\end{array}\right) \zeta(2 k) \zeta(m-2 k)+\frac{1-(-1)^{p}}{2} \zeta(p) \zeta(q) \\
& +(-1)^{p} \sum_{k=1}^{[q / 2]}\left(\begin{array}{c}
m-2 k-1 \\
p-1
\end{array}\right) \zeta(2 k) \zeta(m-2 k)
\end{aligned}
$$

where $\zeta(1)$ should be interpreted as 0 wherever it occurs.

Theorem 3.2. For an odd weight $m=3 l$ ( $l$ is a positive integer), the Euler sums are reducible to zeta values,

$$
\begin{aligned}
\sum_{n=1}^{\infty} \frac{\zeta_{n}^{2}(l)}{n^{l}}= & \frac{1}{3} \zeta^{3}(l)+\zeta(3 l)\left(\frac{1}{6}-\frac{(-1)^{l}}{2}\left(\begin{array}{c}
3 l-1 \\
l
\end{array}\right)-\frac{(-1)^{l}}{2}\left(\begin{array}{c}
3 l-1 \\
2 l
\end{array}\right)\right) \\
& +(-1)^{l} \sum_{k=1}^{[l / 2]}\left(\begin{array}{c}
3 l-2 k-1 \\
2 l-1
\end{array}\right) \zeta(2 k) \zeta(3 l-2 k)+\frac{1-(-1)^{l}}{2} \zeta(l) \zeta(2 k) \\
& +(-1)^{l} \sum_{k=1}^{l}\left(\begin{array}{c}
3 l-2 k-1 \\
l-1
\end{array}\right) \zeta(2 k) \zeta(3 l-2 k) .
\end{aligned}
$$

Proof. Taking $p=l, q=2 l$ in (3.1) and combining (1.5) gives (3.2).

Taking $l=2$ in (1.5) and $l=3$ in (3.2), we have

$$
\sum_{n=1}^{\infty} \frac{\zeta_{n}^{2}(2)}{n^{2}}=\sum_{n=1}^{\infty} \frac{\zeta_{n}(2)}{n^{4}}+\frac{1}{3}\left\{\zeta^{3}(2)-\zeta(6)\right\},
$$




$$
\sum_{n=1}^{\infty} \frac{\zeta_{n}^{2}(3)}{n^{3}}=\frac{253}{6} \zeta(9)+\frac{1}{3} \zeta^{3}(3)-21 \zeta(2) \zeta(7)-6 \zeta(4) \zeta(5) .
$$

From [7], we obtain

$$
\sum_{n=1}^{\infty} \frac{\zeta_{n}(2)}{n^{4}}=-\frac{1}{3} \zeta(6)+\zeta^{2}(3) .
$$

Combining (3.3)(3.5) gives $(3.3)^{\prime}$ :

$$
\sum_{n=1}^{\infty} \frac{\zeta_{n}^{2}(2)}{n^{2}}=\frac{19}{24} \zeta(6)+\zeta^{2}(3)
$$

In their paper, "Euler Sums and Contour Integral Representations", Philippe Flajolet and Bruno Salvy gave the following conclusion: If $p_{1}+p_{2}+q$ is even, and $p_{1}>1, p_{2}>1, q>1$, the quadratic sums

$$
S_{p_{1} p_{2}, q}=\sum_{n=1}^{\infty} \frac{\zeta_{n}\left(p_{1}\right) \zeta_{n}\left(p_{2}\right)}{n^{q}}
$$

are reducible to linear sums (see Theorem 4.2 in the reference [5]). Hence we obtain from (2.6) and Theorem 4.2 in the reference [5] the following theorem:

Theorem 3.3. For integer $l>1$, the cubic sums

$$
S_{l^{3}, l}=\sum_{n=1}^{\infty} \frac{\zeta_{n}^{3}(l)}{n^{l}}
$$

are reducible to linear sums.

From Theorem 4.2 in the reference [5], let $p_{1}=p_{2}=2, q=4$. After a little simplification, we deduce that

$$
\sum_{n=1}^{\infty} \frac{\zeta_{n}^{2}(2)}{n^{4}}=11 S_{2,6}+\frac{457}{18} \zeta(8)+6 \zeta(2) \zeta^{2}(3)-40 \zeta(3) \zeta(5),
$$

where $S_{2,6}=\sum_{n=1}^{\infty} \frac{\zeta_{n}(2)}{n^{6}}$. Substituting (3.6) into (2.6) respectively, we obtain

$$
\sum_{n=1}^{\infty} \frac{\zeta_{n}^{3}(2)}{n^{2}}=\frac{31}{2} S_{2,6}+\frac{3855}{96} \zeta(8)+9 \zeta(2) \zeta^{2}(3)-60 \zeta(3) \zeta(5)
$$

In [5], Philippe Flajolet and Bruno Salvy proves (3.8),

$$
\begin{aligned}
\sum_{n=1}^{\infty} \frac{L_{n}(1)}{n^{2}}= & \frac{\pi^{2}}{4} \ln 2-\frac{1}{4} \zeta(3), \quad \sum_{n=1}^{\infty} \frac{H_{n}(-1)^{n-1}}{n^{2}}=\frac{5}{8} \zeta(3) \\
& \sum_{n=1}^{\infty} \frac{L_{n}(1)(-1)^{n-1}}{n^{2}}=\frac{\pi^{2}}{4} \ln 2-\frac{5}{8} \zeta(3)
\end{aligned}
$$


It is easily seen that

$$
\begin{aligned}
\sum_{n=1}^{\infty} \frac{\zeta_{n}(m)(-1)^{n-1}}{n^{s}} & +\sum_{n=1}^{\infty} \frac{L_{n}(s)}{k^{m}} \\
= & \left(1-\frac{1}{2^{s-1}}\right) \zeta(m) \zeta(s)+\left(1-\frac{1}{2^{m+s-1}}\right) \zeta(m+s), \\
\sum_{n=1}^{\infty} \frac{L_{n}(m)(-1)^{n-1}}{n^{s}} & +\sum_{n=1}^{\infty} \frac{L_{n}(s)(-1)^{k-1}}{k^{m}} \\
& =\left(1-\frac{1}{2^{m-1}}\right)\left(1-\frac{1}{2^{s-1}}\right) \zeta(m) \zeta(s)+\zeta(m+s) .
\end{aligned}
$$

Substituting (3.8) into (3.9)(3.10), we get

$$
\begin{aligned}
& \sum_{n=1}^{\infty} \frac{\zeta_{n}(2)(-1)^{n-1}}{n}=\zeta(3)-\frac{\pi^{2}}{12} \ln 2, \\
& \sum_{n=1}^{\infty} \frac{L_{n}(2)(-1)^{n-1}}{n}=\frac{13}{8} \zeta(3)-\frac{\pi^{2}}{6} \ln 2 .
\end{aligned}
$$

Taking $x=-1$ in Corollary 2.9, we have

$$
\ln ^{3} 2=\sum_{n=1}^{\infty} \frac{\zeta_{n}(2)-H_{n}^{2}}{n+1}(-1)^{n-1} .
$$

Combining (3.11) and (3.12), we obtain

$$
\sum_{n=1}^{\infty} \frac{H_{n}^{2}(-1)^{n-1}}{n}=\frac{3}{4} \zeta(3)+\frac{1}{3}(\ln 2)^{3}-\frac{\pi^{2}}{12} \ln 2 .
$$

Taking $l=1$ in (1.6) and combining (3.8), we have

$$
\sum_{n=1}^{\infty} \frac{L_{n}^{2}(1)(-1)^{n-1}}{n}=\frac{1}{3} \ln ^{3} 2+\frac{\pi^{2}}{4} \ln 2-\frac{1}{2} \zeta(3) .
$$

Taking $m=4,5$ and $x=-1$ in (2.12), we obtain

$$
\begin{aligned}
\ln ^{4}(2)= & \zeta(4)+4 \sum_{n=1}^{\infty} \frac{L_{n}(1)(-1)^{n}}{(n+1)^{3}} \\
& +6 \sum_{n=1}^{\infty} \frac{L_{n}^{2}(1)}{(n+1)^{2}}+4 \sum_{n=1}^{\infty} \frac{L_{n}^{3}(1)(-1)^{n}}{(n+1)} \\
\ln ^{5}(2)= & \frac{15}{16} \zeta(5)+5 \sum_{n=1}^{\infty} \frac{L_{n}(1)}{(n+1)^{4}}+10 \sum_{n=1}^{\infty} \frac{L_{n}^{2}(1)(-1)^{n}}{(n+1)^{3}} \\
& +10 \sum_{n=1}^{\infty} \frac{L_{n}^{3}(1)}{(n+1)^{2}}+5 \sum_{n=1}^{\infty} \frac{L_{n}^{4}(1)(-1)^{n}}{(n+1)} .
\end{aligned}
$$


Similarly to Theorem 2.1, constructing function $y=\sum_{n=1}^{\infty} L_{n}^{2}(1) \zeta_{n}(2) x^{n}, y=$ $\sum_{n=1}^{\infty} L_{n}^{2}(1) L_{n}(2) x^{n}$, we have

$$
\begin{aligned}
\zeta(4)-\zeta(2) \ln ^{2} 2= & \sum_{n=1}^{\infty} \frac{-L_{n}^{2}(1)}{(n+1)^{2}}-2 \sum_{n=1}^{\infty} \frac{(-1)^{n} L_{n}(1) \zeta_{n}(2)}{(n+1)} \\
& -2 \sum_{n=1}^{\infty} \frac{(-1)^{n} L_{n}(1)}{(n+1)^{3}}+\sum_{n=1}^{\infty} \frac{-\zeta_{n}(2)}{(n+1)^{2}}, \\
\frac{1}{2} \zeta(2) \ln ^{2} 2-\frac{7}{8} \zeta(4)= & \sum_{n=1}^{\infty} \frac{L_{n}^{2}(1)(-1)^{n}}{(n+1)^{2}}+\sum_{n=1}^{\infty} \frac{2(-1)^{n} L_{n}(1) L_{n}(2)}{(n+1)} \\
& +\sum_{n=1}^{\infty} \frac{2 L_{n}(1)}{(n+1)^{3}}+\sum_{n=1}^{\infty} \frac{L_{n}(2)}{(n+1)^{2}} .
\end{aligned}
$$

From [5], Philippe Flajolet and Bruno Salvy gave the following formula (see Theorem 7.1 in the reference [5])

$$
\begin{aligned}
\sum_{n=1}^{\infty} \frac{L_{n}(1)}{n^{s}}= & \zeta(s) \ln 2-\frac{s}{2} \zeta(s+1) \\
& +L(s+1)+\frac{1}{2} \sum_{j=1}^{s} L(s-j+1) L(j), \quad 1<s \in Z .
\end{aligned}
$$

where $L(s)=\sum_{n=1}^{\infty} \frac{(-1)^{n-1}}{n^{s}}=\left(1-2^{1-s}\right) \zeta(s)$. Let $s=3,4$ in $(3.19)$, we get

$$
\begin{aligned}
& \sum_{n=1}^{\infty} \frac{L_{n}(1)}{n^{3}}=\frac{7}{4} \zeta(3) \ln 2-\frac{\pi^{4}}{288}, \\
& \sum_{n=1}^{\infty} \frac{L_{n}(1)}{n^{4}}=\frac{15}{8} \ln 2 \zeta(4)+\frac{3}{8} \zeta(2) \zeta(3)-\frac{17}{16} \zeta(5) .
\end{aligned}
$$

In [1], David H. Bailey, Jonathan M. Borwein and Roland Girgensohn gave the following formula by the experimental method (see Table 3 in the reference [1])

$$
\begin{aligned}
\sum_{n=1}^{\infty} \frac{L_{n}^{2}(1)}{(n+1)^{2}} & =6 L i_{4}\left(\frac{1}{2}\right)+\frac{1}{4} \ln ^{4} 2-\frac{29}{8} \zeta(4)+\frac{3}{2} \zeta(2) \ln ^{2} 2, \\
\sum_{n=1}^{\infty} \frac{L_{n}^{2}(1)(-1)^{n-1}}{(n+1)^{2}} & =\frac{7}{4} \zeta(3) \ln 2+\frac{37 \pi^{4}}{1440}-\frac{\pi^{2} \ln ^{2} 2}{3}-\frac{1}{6} \ln ^{4} 2-4 L i_{4}\left(\frac{1}{2}\right), \\
\sum_{n=1}^{\infty} \frac{L_{n}^{2}(1)}{(n+1)^{3}} & =4 L i_{5}\left(\frac{1}{2}\right)-\frac{1}{30} \ln ^{5} 2-\frac{17}{32} \zeta(5)-\frac{11}{8} \zeta(4) \ln 2 \\
& +\frac{7}{4} \zeta(3) \ln ^{2} 2+\frac{1}{3} \zeta(2) \ln ^{3} 2-\frac{3}{4} \zeta(2) \zeta(3),
\end{aligned}
$$




$$
\begin{aligned}
\sum_{n=1}^{\infty} \frac{L_{n}^{2}(1)(-1)^{n-1}}{(n+1)^{3}}= & 4 \ln 2 L i_{4}\left(\frac{1}{2}\right)+\frac{1}{6} \ln ^{5} 2-\frac{79}{32} \zeta(5)+\frac{11}{8} \zeta(4) \ln 2 \\
& -\zeta(2) \ln ^{3} 2+\frac{3}{8} \zeta(2) \zeta(3)
\end{aligned}
$$

From [2] we know that

$$
\sum_{n=1}^{\infty} \frac{L_{n}(1)(-1)^{n-1}}{n^{3}}=\frac{\pi^{4}}{60}+\frac{\pi^{2} \ln ^{2} 2}{12}-\frac{1}{12} \ln ^{4} 2-2 L i_{4}\left(\frac{1}{2}\right)
$$

Combining (3.15)-(3.18) and (3.20)-(3.26), we obtain

$$
\begin{aligned}
\sum_{n=1}^{\infty} \frac{L_{n}^{3}(1)(-1)^{n-1}}{(n+1)}= & \frac{11 \pi^{2} \ln ^{2} 2}{24}+\frac{\ln ^{4} 2}{24}+7 L i_{4}\left(\frac{1}{2}\right)-\frac{15 \pi^{4}}{288} \\
\sum_{n=1}^{\infty} \frac{(-1)^{n-1} L_{n}(1) L_{n}(2)}{(n+1)}= & \frac{5}{16} \zeta(4)-\frac{7}{8} \zeta(3) \ln 2-2 \zeta(2) \ln ^{2} 2 \\
\sum_{n=1}^{\infty} \frac{(-1)^{n-1} L_{n}(1) \zeta_{n}(2)}{(n+1)}= & \frac{3}{4} \zeta(2)(\ln 2)^{2}+\frac{1}{24}(\ln 2)^{4} \\
& +L i_{4}\left(\frac{1}{2}\right)-\frac{7}{16} \zeta(4) \\
\sum_{n=1}^{\infty} \frac{L_{n}^{4}(1)(-1)^{n-1}}{(n+1)}= & \frac{11}{30} \ln ^{5} 2-4 \ln 2 L i_{4}\left(\frac{1}{2}\right)-\frac{73}{2} \zeta(4) \ln 2+7 \zeta(2) \ln ^{3} 2 \\
& +\frac{5}{8} \zeta(2) \zeta(3)+\frac{709}{16} \zeta(5)-48 L i_{5}\left(\frac{1}{2}\right)
\end{aligned}
$$

we have

Similarly, constructing function $y=\sum_{n=1}^{\infty} L_{n}^{2}(1) \zeta_{n}(3) x^{n}, y=\sum_{n=1}^{\infty} L_{n}^{2}(1) L_{n}(3) x^{n}$,

$$
\begin{aligned}
\zeta(5)-\zeta(3) \ln ^{2} 2= & 2 \sum_{n=1}^{\infty} \frac{(-1)^{n-1} L_{n}(1) \zeta_{n}(3)}{(n+1)}+2 \sum_{n=1}^{\infty} \frac{(-1)^{n-1} L_{n}(1)}{(n+1)^{4}} \\
& -\sum_{n=1}^{\infty} \frac{L_{n}^{2}(1)}{(n+1)^{3}}-\sum_{n=1}^{\infty} \frac{\zeta_{n}(3)}{(n+1)^{2}}, \\
\frac{15}{16} \zeta(5)-\frac{3}{4} \zeta(3) \ln ^{2} 2= & 2 \sum_{n=1}^{\infty} \frac{(-1)^{n-1} L_{n}(1) L_{n}(3)}{(n+1)}-2 \sum_{n=1}^{\infty} \frac{L_{n}(1)}{(n+1)^{4}} \\
& +\sum_{n=1}^{\infty} \frac{(-1)^{n-1} L_{n}^{2}(1)}{(n+1)^{3}}-\sum_{n=1}^{\infty} \frac{L_{n}(3)}{(n+1)^{2}} .
\end{aligned}
$$


From Theorem 7.2 and 7.2 in the reference [5], we obtain

$$
\begin{aligned}
\sum_{n=1}^{\infty} \frac{(-1)^{n-1} L_{n}(1)}{n^{4}} & =\frac{15}{8} \zeta(4) \ln 2+\frac{3}{4} \zeta(2) \zeta(3)-\frac{59}{32} \zeta(5), \\
\sum_{n=1}^{\infty} \frac{L_{n}(3)}{n^{2}} & =\frac{1}{8} \zeta(2) \zeta(3)+\frac{41}{32} \zeta(5) .
\end{aligned}
$$

Let $p=3, q=2$ in lemma 3.1 , we get

$$
\sum_{n=1}^{\infty} \frac{\zeta_{n}(3)}{n^{2}}=\frac{11}{2} \zeta(5)-2 \zeta(2) \zeta(3) .
$$

Combining (3.21)(3.24)(3.25)(3.31)-(3.34) gives

$$
\begin{aligned}
\sum_{n=1}^{\infty} \frac{L_{n}(1) \zeta_{n}(3)(-1)^{n-1}}{(n+1)}= & 2 L i_{5}\left(\frac{1}{2}\right)-\frac{1}{60} \ln ^{5} 2-\frac{23}{64} \zeta(5)+\frac{19}{16} \zeta(4) \ln 2 \\
& +\frac{3}{8} \zeta(3) \ln ^{2} 2+\frac{1}{6} \zeta(2) \ln ^{3} 2-\frac{5}{8} \zeta(2) \zeta(3) \\
\sum_{n=1}^{\infty} \frac{L_{n}(1) L_{n}(3)(-1)^{n-1}}{(n+1)}= & -2 \ln 2 L i_{4}\left(\frac{1}{2}\right)-\frac{1}{12} \ln ^{5} 2+\frac{19}{16} \zeta(4) \ln 2 \\
& +\frac{1}{2} \zeta(2) \ln ^{3} 2+\frac{1}{4} \zeta(2) \zeta(3)-\frac{1}{8} \zeta(5)-\frac{3}{8} \zeta(3) \ln ^{2} 2
\end{aligned}
$$

Acknowledgements. The authors will be very grateful to the referee for his/her valuable suggestions.

\section{References}

[1] D.H. Bailey, J.M. Borwein, and R. Girgensohn, Experimental evaluation of Euler sums, Experimental Mathematics 3(1) (1994), 17-30.

[2] D. Borwein, J.M. Borwein, and R. Girgensohn, Explicit evaluation of Euler sums, Proc. Edinburgh Math. 38 (1995), 277-294.

[3] J. Borwein, P. Borwein, R. Girgensohn, and S. Parnes, A discussion, Experimental Mathematics., 1995.

[4] M. Eie, C.-S. Wei, Evaluations of some quadruple Euler sums of even weight, Functiones et Approximatio 46(1) (2012), 63-67.

[5] P. Flajolet and B. Salvy, Euler sums and contour integral representations, Experimental Mathematics 7(1) (1998), 15-35.

[6] L. Comtet, Advanced Combinatorics, Boston: D Reidel Publishing Company, 1974.

[7] P. Sun, The 6-order sums of Riemann zeta function, Acta Mathematica Sinica, chinese Series ., 50(2) (2007), 373-384.

Address: Ce Xu, Jinfa Cheng: School of Mathematical Sciences, Xiamen University, Fujian 361005, P. R. China.

E-mail: xuce1242063253@163.com,jfcheng@xmu.edu.cn

Received: 12 March 2015; revised: 19 September 2015 